\title{
Posterior Reversible Encephalopathy Syndrome in a Patient of Sepsis-induced Cardiomyopathy, Successfully Managed with Intra-aortic Balloon Pump
}

\author{
Anudeep Saxena ${ }^{1}$, Vivek Bhargava ${ }^{2}$, Aditya Shreya $^{3}$, Anshul Patodia $^{4}$, Pradeep Goyal ${ }^{5}$
}

\begin{abstract}
In patients with septic shock refractory to pharmacological agents, mechanical devices have been used successfully, although the reports are scarce. We report a case of septic shock where intra-aortic balloon pump (IABP) initiation leads to drastic improvement and survival from severe septic cardiomyopathy when conventional therapy was not effective. A 19-year-old male patient underwent surgery for adenocarcinoma descending colon. On day 8 he was reoperated for anastomotic leak and developed severe cardiomyopathy associated with septic shock, postoperatively. When he was in a vicious cycle of refractory hypotension, metabolic acidosis and severe cardiomyopathy, IABP was instituted along with other management for septic shock. Over next 3 days patient's hemodynamics improved and IABP was weaned off. While recovering from shock he developed posterior reversible encephalopathy syndrome which was promptly managed. This case report emphasizes on early institution of IABP in case of severe left ventricular dysfunction in septic shock.

Keywords: Intra-aortic balloon pump, Posterior reversible encephalopathy syndrome, Septic cardiomyopathy

Indian Journal of Critical Care Medicine (2019): 10.5005/jp-journals-10071-23152
\end{abstract}

\section{INTRODUCTION}

Sepsis-induced cardiomyopathy is a complication of severe sepsis and septic shock described as a reversible myocardial depression that occurs in patients with septic shock. Sepsis-induced cardiomyopathy has three characteristics: left ventricular dilatation, depressed ejection fraction, and recovery in 7-10 days. Incidence of sepsis-induced left ventricular hypokinesia has been reported to be as high as $60 \%{ }^{2}$ Use of IABP has been reported to be lifesaving in certain sepsis-induced cardiomyopathy. ${ }^{3,4}$ We report a case with $I A B P$ introduction leading to drastic improvement, and survival from severe septic cardiomyopathy when conventional therapy was not effective, also how the course in the hospital was complicated by posterior reversible encephalopathy syndrome (PRES).

\section{Case Report}

A 19-year-old Indian male was admitted with complains of abdominal pain, nausea, vomiting, and weight loss. He was diagnosed with moderately differentiated adenocarcinoma (T2NOMO) and underwent left hemicolectomy, colocolic, and jejunojejunal anastomosis. Seven days postoperatively patient developed anastomotic leak for which he was reoperated. Postoperatively (day 2 of ICU), patient required high dose vasopressor, his condition worsened and had to be put on mechanical ventilation for pulmonary edema (Fig. 1). 2D-echocardiography revealed severely depressed left ventricular function.

Echo findings are as follows: Global hypokinesia more in anteroseptal wall, anterior wall, and intraventricular septum. Left ventricular ejection fraction (LVEF) is $15-20 \%$ (visually estimated). Mild mitral regurgitation, mild tricuspid regurgitation, no pulmonary artery hypertension, intra-ventricular and intra-atrial septum are intact. No pericardial effusion, clot or vegetation seen. $\overline{\text { 1,2Department of Anesthesiology, Narayana Multispeciality Hospital, }}$ Jaipur, Rajasthan, India.

${ }^{3}$ Department of Gastrointestinal Surgery, Narayana Multispeciality Hospital, Jaipur, Rajasthan, India.

${ }^{4}$ Department of Cardiology, Narayana Multispeciality Hospital, Jaipur, Rajasthan, India.

${ }^{5}$ Department of Cardiac Anesthesiology, Narayana Multispeciality Hospital, Jaipur, Rajasthan, India.

Corresponding Author: Anudeep Saxena, Department of Anesthesiology, Narayana Multispeciality Hospital, Jaipur, Rajasthan, India, e-mail: dranudeepsaxena@yahoo.in

How to cite this article: Saxena A, Bhargava V, et al. Posterior Reversible Encephalopathy Syndrome in a Patient of Sepsisinduced Cardiomyopathy, Successfully Managed with Intra-aortic Balloon Pump. Indian J Crit Care Med 2019;23(4):188-190.

Source of support: Nil

Conflict of interest: None

ECG showed sinus tachycardia (heart rate-150 bpm), poor R wave progression and nonspecific ST-T changes. Troponin I 1.69 $\mathrm{ng} / \mathrm{mL}$, CPK-MB $5.2 \mathrm{ng} / \mathrm{mL}$, BNP $2000 \mathrm{pg} / \mathrm{mL}$.

Possibility of acute coronary syndrome was kept as a differential, but possibility of septic myocarditis was strongly considered. Patient was started on daily aspirin $75 \mathrm{mg}$, clopidogrel $75 \mathrm{mg}$, injection fondaparinux $2.5 \mathrm{mg}$, and injection torsemide $20 \mathrm{mg}$ thrice daily.

Over the next 24 hours (day 3) patient's hypotension and metabolic acidosis worsened despite high dosage of epinephrine, norepinephrine and dobutamine as well as stress dose of hydrocortisone. His tachycardia was bothersome. He had chilled peripheries and seemed to be maximally vasoconstricted. His urine output started falling. Antibiotics were escalated according to pus culture sensitivity reports. Considering cardiogenic shock to be

(c) The Author(s). 2019 Open Access This article is distributed under the terms of the Creative Commons Attribution 4.0 International License (http://creativecommons. org/licenses/by/4.0/), which permits unrestricted use, distribution, and non-commercial reproduction in any medium, provided you give appropriate credit to the original author(s) and the source, provide a link to the Creative Commons license, and indicate if changes were made. The Creative Commons Public Domain Dedication waiver (http://creativecommons.org/publicdomain/zero/1.0/) applies to the data made available in this article, unless otherwise stated. 


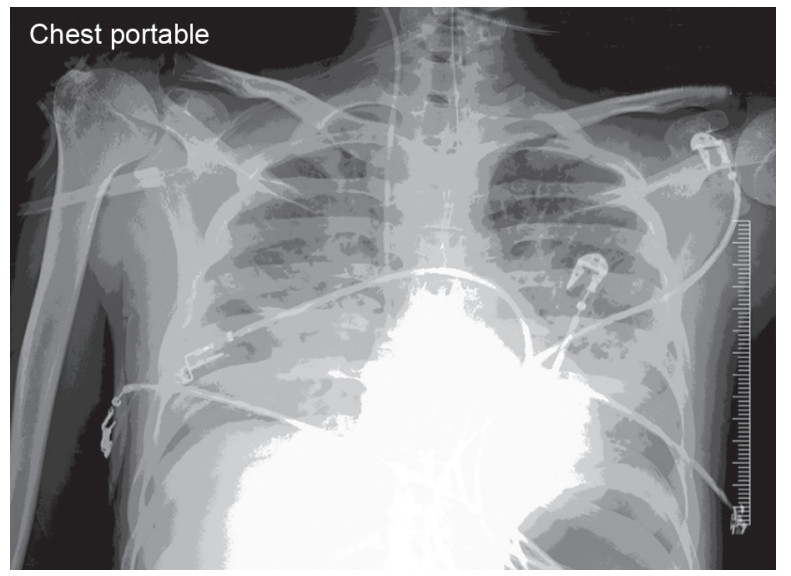

Fig. 1: X-ray chest of patient on day 2 of intensive care unit (ICU) admission when he developed pulmonary edema.

primarily responsible for the patient's condition, it was planned to put IABP. Without wasting time $34 \mathrm{Fr}$ IABP catheter was inserted through right femoral artery. IABP support was initiated with the use of 1:1 electrocardiographic triggering. In few hours CVP was reduced from $18 \mathrm{~mm} \mathrm{Hg}$ to $10 \mathrm{~mm} \mathrm{Hg}$ and heart rate settled from $160 \mathrm{bpm}$ to $120 \mathrm{bpm}$.

Over next 48 hours patient's hemodynamic improved. Lungs were clearer (Fig. 2). Heart rate started settling from $120 \mathrm{bpm}$ to around $100 \mathrm{bpm}$. Ionotropic supports were gradually tapered

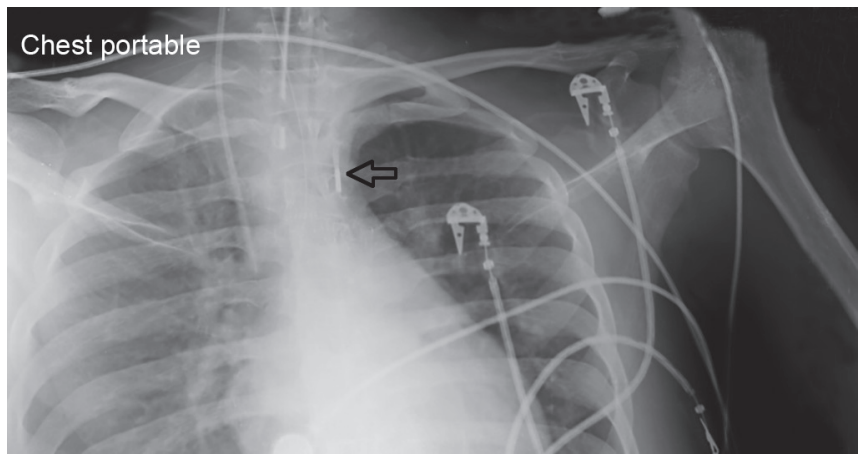

Fig. 2: X-ray chest of patient on day 6. Intra-aortic balloon pump can be seen just distal to arch of aorta. Clearing of pulmonary edema as compared to the previous X-ray can be noted.

maintaining a mean arterial pressure of $65 \mathrm{~mm} \mathrm{Hg}$. Patient started pouring good urine output. On day 6 (day 3 of starting IABP), platelet counts started falling. Patient was maintaining his blood pressure at minimum dose of epinephrine and norepinephrine. IABP was removed. Till next day, when the effect of atracurium was completely weaned off, patient did not become alert. He was opening eyes spontaneously but was not following command; gaze was upward, bilateral extensor planter reflex. MRI brain was done which showed symmetrical mild ill-defined hyperintensity in bilateral occipital lobes, basal ganglia-thalamic region and splenium of corpus callosum (Fig. 3). This was suggestive of possibility of PRES.
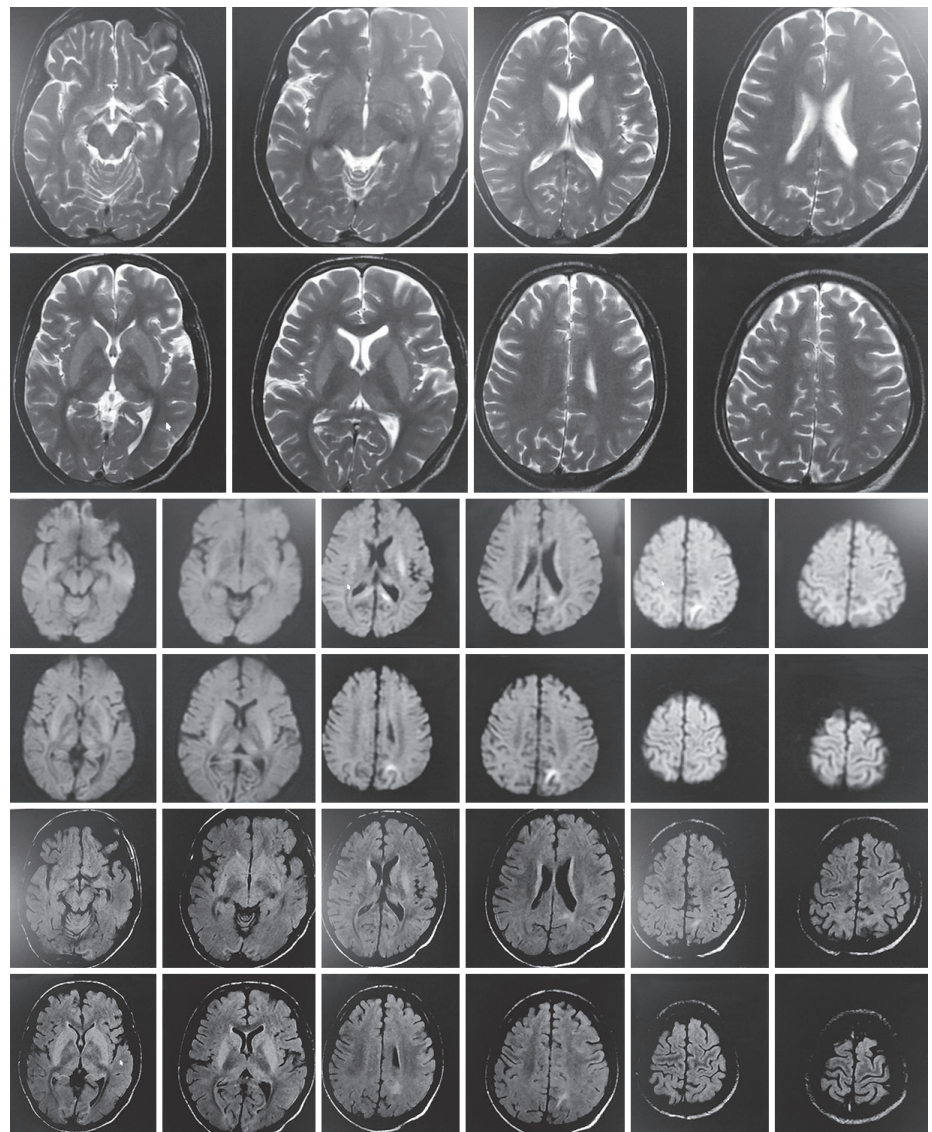

Fig. 3: There is presence of fairly bilaterally symmetrical, ill defined, mild T2, and FLAIR hyperintensity in bilateral basal ganglia and thalamus. In the splenium of corpus callosum and in the cortical-subcortical aspect of bilateral occipital lobes and left posterior parietal lobe there is mild DWI hyperintensity with corresponding mild hypointensity on ADC images (predominantly in the splenium of corpus callosum and in subcortical aspect of left posterior parietal lobe), suggesting diffusion restriction. 
On day 9 of ICU admission patient's tracheostomy was done. Patient continued to be on vasopressor and inotropes, although requirement was low. Antibiotics were further modified according to blood and other fluid culture reports. Over next 5 days epinephrine and norepinephrine were completely withdrawn. Patient neurologically improved and started following commands. After pressure support trial for a day, on day 16 of ICU admission his ventilator support was withdrawn. On day 18 , patient's repeat 2D echo was performed which showed an improved LVEF of $40-45 \%$ with mild septal hypokinesia. He was shifted out of ICU.

His trachea was decannulated and on day 21 he was discharged from the hospital with functioning colostomy in situ.

\section{DISCUSSION AND CONCLUSION}

In sepsis, both the peripheral oxygen requirement and cardiac output are generally increased. However, in patients with septic cardiomyopathy, the cardiac output becomes inadequate to supply the increased systemic and myocardial oxygen requirement. Metabolic acidosis further impairs myocardial contractility; patient enters in a vicious circle and eventually succumbs. The two primary benefits of IABP are augmented coronary perfusion and reduced LV afterload thereby increased cardiac output.

Our idea of initiating IABP was to buy time for the antibiotics to start acting. So that the oxygen requirement of the patient comes down to normal, that could be sustained by a compromised heart.

The time of initiating IABP is particularly important as shown previously by Ogunbayo $\mathrm{G}$ et al. ${ }^{5}$ They have concluded that early use of IABP (within 24 hours of presentation) appears to offer a mortality benefit. In our patient we initiated IABP within 12 hours of identification of cardiomyopathy and LV dysfunction.

Posterior reversible encephalopathy syndrome is commonly, but not always associated with acute hypertension. ${ }^{6}$ In a retrospective study, Bartynski et al. reported an association of PRES with infection, sepsis and shock in $26.3 \%$ (25 out of 106) patients. ${ }^{7}$
In our patient there was no episode of hypertension. In sepsis, an alteration in vascular tone develops secondary to competing vasoconstrictive (platelet degranulation with thromboxane release, endothelin-1, angiotensin, vasopressin, and central sympathetic stimulation) and vasodilatory (nitric oxide, prostacyclin) effects. ${ }^{8,9}$

We suggest that in a patient of septic shock with cardiomyopathy, if pharmacologic agents are insufficient in maintaining systemic perfusion, then IABP should be considered promptly, particularly in patients with severe LV dysfunction (LVF $<30 \%)$. Further elaborated trials are required before these mechanical devices would probably establish their place in future sepsis guidelines.

\section{References}

1. Sato R, Nasu M. A review of sepsis-induced cardiomyopathy. J Intensive Care 2015;3:48.

2. Vieillard-Baron A, Caille V, et al. Actual incidence of global left ventricular hypokinesia in adult septic shock. Crit Care Med 2008;36:1701-1706.

3. Nakamura K, Doi K, et al. Endotoxin adsorption by polymyxin B column or intra-aortic balloon pumping use for severe septic cardiomyopathy. Am J Emerg Med 2013;31:893. e1-3.

4. Hiromi T, Toida C, et al. Two cases with intra-aortic balloon pumping use for severe septic cardiomyopathy. Acute Med Surg 2017;4:446-450.

5. Ogunbayo $\mathrm{G}$, Olorunfemi $\mathrm{O}$, et al. Outcomes of intra-aortic balloon pump use in myocarditis complicated by cardiogenic shock. J Am Coll Cardiol 2017;69:860.

6. McKinney AM, Short J, et al. Posterior reversible encephalopathy syndrome: incidence of atypical regions of involvement and imaging findings. Am J Roentgenol 2007;189:904-912.

7. Bartynski WS, Boardman JF, et al. Posterior reversible encephalopathy syndrome in infection, sepsis, and shock. Am J Neuroradiol 2006;27:2179-2190.

8. Munford RS. Sepsis, severe sepsis, and septic shock. In: Mandell GL, Bennett JE, Dolin R (Eds). Principles and Practice of Infectious Disease. Philadelphia: Elsevier; 2005. pp. 906-926.

9. Symeonides S, Balk RA. Nitric oxide in the pathogenesis of sepsis. Infect Dis Clin North Am 1999;13:449-463, x. 